\title{
Bilevel Programming for Generating Discrete Representations in Multiobjective Optimization ${ }^{* \dagger}$
}

\author{
Gokhan Kirlik \\ Department of Radiation Oncology, University of Maryland \\ School of Medicine, Baltimore, Maryland 21201 \\ gokhankirlik@umm.edu \\ Serpil Sayın $\ddagger$ \\ College of Administrative Sciences and Economics \\ Koç University, Sariyer, Istanbul, 34450, Turkey \\ ssayin@ku.edu.tr
}

\begin{abstract}
The solution to a multiobjective optimization problem (MOP) consists of the nondominated set that portrays all relevant trade-off information. The ultimate goal is to identify a Decision Maker's most preferred solution without generating the entire set of nondominated solutions. We propose a bilevel programming formulation that can be used to this end. The bilevel program is capable of delivering an efficient solution that maps into a given set, provided that one exits. If the Decision Maker's preferences are known a priori, they can be used to specify the given set. Alternatively, we propose a method to obtain a representation of the nondominated set when the Decision Maker's preferences are not available. This requires a thorough search of the outcome space. The search can be facilitated by a partitioning scheme similar to the ones used in global optimization. Since the bilevel programming formulation either finds a nondominated solution in a given partition element or determines that there is none, a representation with a specified coverage error level can be found in a finite number of iterations. While building a discrete representation, the algorithm also generates an approximation of the nondominated set within the specified error factor. We illustrate the algorithm on the multiobjective linear programming problem.
\end{abstract}

Keywords: Multiobjective optimization, Decision Maker, Representation, Nondominated Set, Coverage error, Bilevel programming problem

\section{Introduction}

Many decision making models take into account a single objective. However, most real life decision making problems are more complex and require considering several conflicting objectives in the decision making process. When a mathematical programming framework is

\footnotetext{
*Published in Mathematical Programming, 169, no. 2 (2018): 585-604.

${ }^{\dagger}$ Supported by TUBITAK (Scientific \& Technical Research Council of Turkey), project no. 112M217.

${ }^{\ddagger}$ Corresponding author
} 
utilized to model such problems, the result is a multiobjective optimization problem (MOP) which is a generalization of traditional single-objective optimization. In multiobjective optimization, the set of efficient solutions is sought instead of an optimal solution. An efficient solution has the property that no improvement in any objective is possible without sacrificing at least in one other objective. The collection of all efficient solutions of an MOP is called the efficient set. This set portrays all relevant trade-off information to a Decision Maker and its image under the objective functions is called the nondominated set [13].

When preferences of the Decision Maker are known a priori, a single objective formulation can replace the multiobjective problem by means of aggregation and scalarization. When the Decision Maker's preferences are allowed to evolve during a process of searching for a most preferred solution, an interactive approach may reconstruct the single objective problem several times until a satisfactory solution is obtained. When there is no information about Decision Maker's preferences, obtaining the entire nondominated set becomes the goal, which leads to a computationally prohibitive task except for some special cases. The computational difficulties encountered by methods that seek the entire efficient set have hindered their applicability in realistic settings. To date, interactive methods remain as viable tools despite the fact that they may not be able to guarantee not missing out a truly most preferred solution. A line of research has emerged that suggests obtaining a finite discrete subset of the nondominated set, usually referred to as a representation, as a solution. As we also propose an approach for finding a representation, we will provide a detailed review of this literature after we briefly look at approaches that are designed to find the entire nondominated set.

In multiobjective linear programming (MOLP) problems, the efficient set can be defined as a union of efficient faces [53]. The exact methods for MOLP aim to generate all extreme efficient solutions or all efficient faces of MOLP (see, for e.g., [4], [12],[40]). In multiobjective discrete optimization (MODO), generating all efficient solutions may be computationally expensive even in relatively small problems $[29,7]$. Finding only extreme supported efficient solutions $[38,36]$ may therefore be considered. Multiobjective mixed integer programming (MOMIP) problems are difficult to solve because of the isolated portions of the nondominated set [50, 45]. For MOMIP problems, characterization of the nondominated set is a major challenge, and the current methods are mostly limited to biobjective problems [45].

An early work on representations is Benson and Sayı's global shooting procedure that seeks to find global representations of an MOP with a compact feasible set by constructing a special simplex that contains the feasible set in objective space [3]. Das and Denis propose the boundary intersection method for finding an evenly distributed set of solutions in the nondominated set [8]. Recently Shao and Ehrgott combine these methods to obtain well distributed nondominated solutions for MOLP [44]. Other methods for finding representations are due to Kim and De Weck for bicriteria nonconvex problems [27] and general MOPs [28], Sylva and Crema [46] and Masin and Bukchin [33] for MOMIP problems and Karasakal and Koksalan for MOLP [26]. All of these approaches aspire to deliver good representations whereas the definition of a good representation is discussed more extensively in other studies.

Different measures have been introduced to quantify the quality of a representation $[41,17]$. One of the frequently used measures is the coverage error. This measure assumes that each element of the nondominated set is represented by a closest element in the repre- 
sentation, the distance between the two is the coverage error for that particular point, and the worst represented element of the nondominated set determines the coverage error for the representation. Several methods have been proposed to generate representations that satisfy some of these quality measures. In [42], Sayın gives a method for MOLP problems to find a discrete representation with a specified coverage error level or the maximum coverage possible given a target cardinality by using a mathematical program. Sayın and Kouvelis $[43,30]$ propose a parametric search algorithm with min-max type subproblems to generate a representation with specified coverage error for biobjective discrete optimization problems. For the same problem, Hamacher et al. generate the representation by using boxes and $\varepsilon$-constraint method [22]. Bokrantz and Forsgren introduce an algorithm to approximate nondominated sets of convex problems [5]. They control the quality of the approximation by using the Hausdorff distance between the outer and the inner approximations.

Another common quality measure is the uniformity of the solutions in the representation. Eichfelder gives a method to generate a representation considering the spacing of the solutions in the set $[15,16]$. Leyffer formulates the problem of finding a maximally uniform representation of the nondominated set with a given number of solutions for convex multiobjective optimization problems as a mathematical program with complementarity constraints [31]. Faulkenberg et al., addressing a similar problem, use a bilevel programming formulation in which the upper level formulation controls spacing and the lower level formulation generates the nondominated solutions [18]. Other methods that focus on the bicriteria problem are due to Pereyra et al. [37] and Vaz et al. [47].

In this study, we propose an approach that is capable of focusing on particular regions of interest in the nondominated set. If there is prior information about the preferences of a Decision Maker and these can be expressed via constraints in a mathematical programming setting, our proposed bilevel programming formulation is capable of returning a nondominated solution from the set, provided that one exists. When preferences are not known a priori, this formulation can be used in a method that generates a representation of the nondominated set with respect to a specified coverage error. To do this, we apply partitioning ideas that have been utilized in global optimization [24] to the image of the feasible set in the outcome space. For each partition element, a bilevel programming based subproblem is solved. Once solved, the subproblem either returns a nondominated solution from the partition element or determines that none exists. Depending on this outcome, the partition element is either subdivided and refined further or eliminated from the search. We show that our proposed algorithm is finite, and the resulting representation satisfies the specified coverage error. Moreover, an approximation of the nondominated set is also obtained.

In the following section, we present general definitions for MOP and present the bilevel programming formulation which is used to find a nondominated solution in a given set. In Section 3, the representation algorithm that is built on the bilevel programming based subproblem and related results are given. In Section 4, the representation algorithm is illustrated on MOLP problems by using rectangles as partition elements. Finally, conclusions are presented in Section 5. 


\section{Definitions and a Bilevel Programming Based Formulation}

In multiobjective optimization, $p$ objective functions $f_{j}: \mathbb{R}^{n} \rightarrow \mathbb{R}$ for all $j \in\{1, \ldots, p\}$ are minimized simultaneously. The feasible set is denoted as $\mathcal{X} \subseteq \mathbb{R}^{n}$. Each feasible solution $x \in \mathcal{X}$ is mapped into its corresponding objective vector $y=f(x)$ and $\mathcal{Y}=\left\{y \in \mathbb{R}^{p}: y=\right.$ $f(x)$ for some $x \in \mathcal{X}$ \} is the set of feasible outcomes in the objective space. We assume that objective functions are continuous and $\mathcal{X}$ is compact. Hence, $\mathcal{Y}$ is also compact. In mathematical terms, MOP is defined as:

$$
\begin{array}{lll}
(\text { MOP }) & \min & f(x)=\left[f_{1}(x), \ldots, f_{p}(x)\right] \\
& \text { s.t. } & x \in \mathcal{X} .
\end{array}
$$

Due to conflicting objectives, MOP is expected to have more than one solution. These solutions are called efficient solutions. In the definition below and thereafter, we use $<$ and $\leq$ not in the vector sense but componentwise, i.e. for $y, z \in \mathbb{R}^{n}, y<(\leq) z$ means $y_{i}<(\leq) z_{i}$ for all $i=1, \ldots, n$.

Definition 1. A solution $x^{*} \in \mathcal{X}$ is called an efficient solution if there exists no feasible solution $x \in \mathcal{X}$ such that $f_{j}(x) \leq f_{j}\left(x^{*}\right)$ for all $j \in\{1, \ldots, p\}$ and there exists $\hat{\jmath} \in\{1, \ldots, p\}$ such that $f_{\hat{\jmath}}(x)<f_{\hat{\jmath}}\left(x^{*}\right)$. For an efficient solution $x^{*}, f\left(x^{*}\right) \in \mathbb{R}^{p}$ is referred to as a nondominated solution in the outcome space.

The set of all efficient solutions for MOP is called the efficient set and is denoted as $\mathcal{X}_{E}$. The image of the efficient set in the objective space is called the nondominated set and is denoted as $\mathcal{Y}_{N}$, i.e. $\mathcal{Y}_{N}=\left\{y \in \mathbb{R}^{p}: y=f(x)\right.$ for some $\left.x \in \mathcal{X}_{E}\right\}$.

We model the problem of finding a nondominated solution in a given closed set $S \subseteq \mathbb{R}^{p}$ as a bilevel programming problem. The upper and lower decision variables are $x \in \mathbb{R}^{n}$ and $z \in \mathbb{R}^{n}$ respectively.

$$
\begin{array}{ll}
(B P P) \quad \min _{x} & f^{U}(x, z)=d(f(x), f(z)) \\
\text { s.t. } & x \in \mathcal{X} \\
& f(x) \in S \\
& \min _{z} \quad f^{L}(z)=w^{T} f(z) \\
& \text { s.t. } \quad z \in \mathcal{X} \\
& \quad f(z) \leq f(x) .
\end{array}
$$

In the above formulation, $d(\cdot, \cdot)$ is a metric defined on $\mathbb{R}^{p}$, and $w \in \mathbb{R}^{p}$ is a given positive weight vector. The feasible set of the bilevel program is

$$
M=\{(x, z): x, z \in \mathcal{X}, f(x) \in S, f(z) \leq f(x)\} .
$$

For any $x \in \mathcal{X}$ and $f(x) \in S$, the rational reaction set for the lower level formulation is

$$
\Omega(x)=\underset{z \in \mathcal{X}}{\arg \min }\left\{w^{T} f(z): f(z)-f(x) \leq 0\right\} .
$$

In BPP, for any $x \in \mathbb{R}^{n}$, the lower level aims to find a solution $z \in \mathcal{X}$ such that all components of $f(z)$ are less than or equal to $f(x)$. In other words, while the upper level 
problem seeks a feasible solution whose image is in $S$, the lower level problem seeks a feasible solution in $\mathcal{X}$ that might dominate it. The goal of BPP is to minimize the distance between the images of the two solutions $x \in \mathcal{X}$ with $f(x) \in S$ and $z \in \Omega(x)$ in the outcome space. In doing so, the subproblem serves as an indicator of existence of nondominated solutions in $S$. Before we build the results that are needed to illustrate this, we note that in a solution to a bilevel program in general, there might be alternative optimal solutions to the lower level problem and the choice among these alternative solutions might impact the upper level objective function. This has led to modeling the reaction of the lower level problem in an optimistic or pessimistic sense. In our case, all lower level alternative optimal solutions have the same image in the outcome space. Since the upper level objective function measures distances in the outcome space, we do not need to emphasize a particular way of modeling the lower level reaction.

Lemma 1. Let $w>0$ and $S \subseteq \mathbb{R}^{p}$ be given. For any $x \in \mathcal{X}$ such that $f(x) \in S, \Omega(x) \neq \emptyset$.

Proof. Let $x^{0} \in \mathcal{X}$ be such that $f\left(x^{0}\right) \in S$. Note that $x^{0}$ is feasible to the lower level problem, i.e. the lower level problem cannot be infeasible as long as the upper level problem is feasible. Since $\mathcal{X}$ is compact and $f$ is continuous, $\min _{z \in \mathcal{X}}\left\{w^{T} f(z): f(z)-f\left(x^{0}\right) \leq 0\right\}$ exists and is achieved at some $z^{0} \in \mathcal{X}$. Thus $\Omega\left(x^{0}\right) \neq \emptyset$ follows.

The above result indicates that BPP is always well-defined and any infeasibility of BPP might stem from the upper level problem. We now explore the relationship between efficiency of an $x \in \mathcal{X}$ and its optimality to BPP.

Proposition 1. Let $w>0$ and $S \subseteq \mathbb{R}^{p}$ be given. For any $x \in \mathcal{X}$ such that $f(x) \in S$, any $z^{*} \in \Omega(x)$ is efficient.

Proof. Let $\bar{x} \in \mathcal{X}$ be such that $f(\bar{x}) \in S$. Pick $z^{*} \in \Omega(\bar{x})$. Suppose, to the contrary, that $z^{*}$ is not efficient. Then $\exists z^{\prime} \in \mathcal{X}$ such that $f\left(z^{\prime}\right) \leq f\left(z^{*}\right)$ with $f_{\hat{\jmath}}\left(z^{\prime}\right)<f_{\hat{\jmath}}\left(z^{*}\right)$ for some $\hat{\jmath} \in\{1, \ldots, p\}$. Then $f\left(z^{\prime}\right) \leq f\left(z^{*}\right) \leq f(\bar{x})$ so that $z^{\prime}$ is feasible to the lower level problem and $w^{T} f\left(z^{\prime}\right)<w^{T} f\left(z^{*}\right)$, which contradicts that $z^{*} \in \Omega(\bar{x})$. Thus we conclude that $z^{*}$ is efficient.

Our lower level problem is closely related to a scalarization studied in [25] where it is shown that for a given $x \in \mathcal{X}$, the scalarization will deliver efficient solutions. Along with Proposition 2, Proposition 1 will lead to key results that follow below.

Proposition 2. Let $w>0$ be given. For a given $S \subseteq \mathbb{R}^{p}$, for any efficient solution with $f\left(z^{*}\right) \in S$, there exists $x^{*} \in \mathcal{X}$ such that $z^{*} \in \Omega\left(x^{*}\right)$.

Proof. Let $z^{*} \in \mathcal{X}_{E}$ be such that $f\left(z^{*}\right) \in S$. Let $x^{*}=z^{*}$. By definition, $\Omega\left(x^{*}\right)=$ $\arg \min _{z \in \mathcal{X}}\left\{w^{T} f(z): f(z)-f\left(x^{*}\right) \leq 0\right\}$. Note that $z^{*} \in \mathcal{X}$ and $f\left(z^{*}\right) \leq f\left(x^{*}\right)$. If $z^{*} \notin \Omega\left(x^{*}\right)$, then $\exists z^{\prime} \in \mathcal{X}, f\left(z^{\prime}\right) \leq f\left(x^{*}\right)=f\left(z^{*}\right)$ and $w^{T} f\left(z^{\prime}\right)<w^{T} f\left(z^{*}\right)$, which would contradict efficiency of $z^{*}$. Thus we conclude that $z^{*} \in \Omega\left(x^{*}\right)$.

Proposition 3. Let $w>0$ be given. For a given set $S \subseteq \mathbb{R}^{p}$, if there exists $\left(x^{*}, z^{*}\right)$ that solves the bilevel programming formulation with $d\left(f\left(x^{*}\right), f\left(z^{*}\right)\right)=0$, then $f\left(x^{*}\right) \in S \cap \mathcal{Y}_{N}$. 
Proof. Let $w>0$ and $S \subseteq \mathbb{R}^{p}$ be given. Let $\left(x^{*}, z^{*}\right)$ be an optimal solution of BPP with $d\left(f\left(x^{*}\right), f\left(z^{*}\right)\right)=0$. Since $d$ is a metric, $d\left(f\left(x^{*}\right), f\left(z^{*}\right)\right)=0$ implies that $f\left(x^{*}\right)=f\left(z^{*}\right)$. Note that $z^{*} \in \Omega\left(x^{*}\right)$ since $\left(x^{*}, z^{*}\right)$ is optimal to BPP. Hence, by Proposition $1, z^{*}$ is an efficient solution and $f\left(z^{*}\right) \in \mathcal{Y}_{N}$. Since $f\left(x^{*}\right)=f\left(z^{*}\right), f\left(x^{*}\right) \in \mathcal{Y}_{N}$. Since $x^{*}$ is feasible to BPP, $f\left(x^{*}\right) \in S$, which implies that $f\left(x^{*}\right) \in S \cap \mathcal{Y}_{N}$.

Theorem 1. For a given closed set $S \subseteq \mathbb{R}^{p}, S \cap \mathcal{Y}_{N}=\emptyset$ if and only if either BPP is infeasible or there exists $\left(x^{*}, z^{*}\right)$ that solves BPP with $d\left(f\left(x^{*}\right), f\left(z^{*}\right)\right)>0$.

Proof. $(\Rightarrow)$ Let $S \subseteq \mathbb{R}^{p}$ be closed. Assume that $S \cap \mathcal{Y}_{N}=\emptyset$. Suppose, to the contrary that BPP is neither infeasible nor the optimal solution satisfies $d\left(f\left(x^{*}\right), f\left(z^{*}\right)\right)>0$. Then $d\left(f\left(x^{*}\right), f\left(z^{*}\right)\right)=0$ must hold. By Proposition 3, $f\left(x^{*}\right) \in S \cap \mathcal{Y}_{N}$ follows, which contradicts the assumption.

$(\Leftarrow)$ Suppose BPP is either infeasible or its optimal solution satisfies $d\left(f\left(x^{*}\right), f\left(x^{*}\right)\right)>0$.

i If BPP is infeasible, by Lemma 1, there exists no $x \in \mathcal{X}$ such that $f(x) \in S$, i.e. $\mathcal{Y} \cap S=\emptyset$, which implies $\mathcal{Y}_{N} \cap S=\emptyset$.

ii An optimal solution $\left(x^{*}, z^{*}\right)$ to BPP exists and $d\left(f\left(x^{*}\right), f\left(z^{*}\right)\right)>0$. Now suppose to the contrary, that $S \cap \mathcal{Y}_{N} \neq \emptyset$. Then there exists $x^{\prime} \in \mathcal{X}_{E}$ such that $f\left(x^{\prime}\right) \in S$. Then $\left(x^{\prime}, z^{\prime}\right)$ where $z^{\prime}=x^{\prime}$ is feasible to the bilevel formulation. Moreover, $d\left(f\left(x^{\prime}\right), f\left(z^{\prime}\right)\right)=$ 0 , which contradicts optimality of $\left(x^{*}, z^{*}\right)$.

Hence we conclude that $S \cap \mathcal{Y}_{N}=\emptyset$ must hold.

To summarize, Theorem 1 states that BPP can be used to identify existence of nondominated solutions in a set. An infeasible BPP implies that $S \cap \mathcal{Y}=\emptyset$. A feasible BPP with a positive optimal objective function value (of the upper level problem) implies that $S \cap \mathcal{Y}_{N}=\emptyset$. A feasible BPP with a zero optimal objective function value implies that $S \cap \mathcal{Y}_{N} \neq \emptyset$ and $x^{*} \in \mathcal{X}$ such that $f\left(x^{*}\right) \in S \cap \mathcal{Y}_{N}$ is returned.

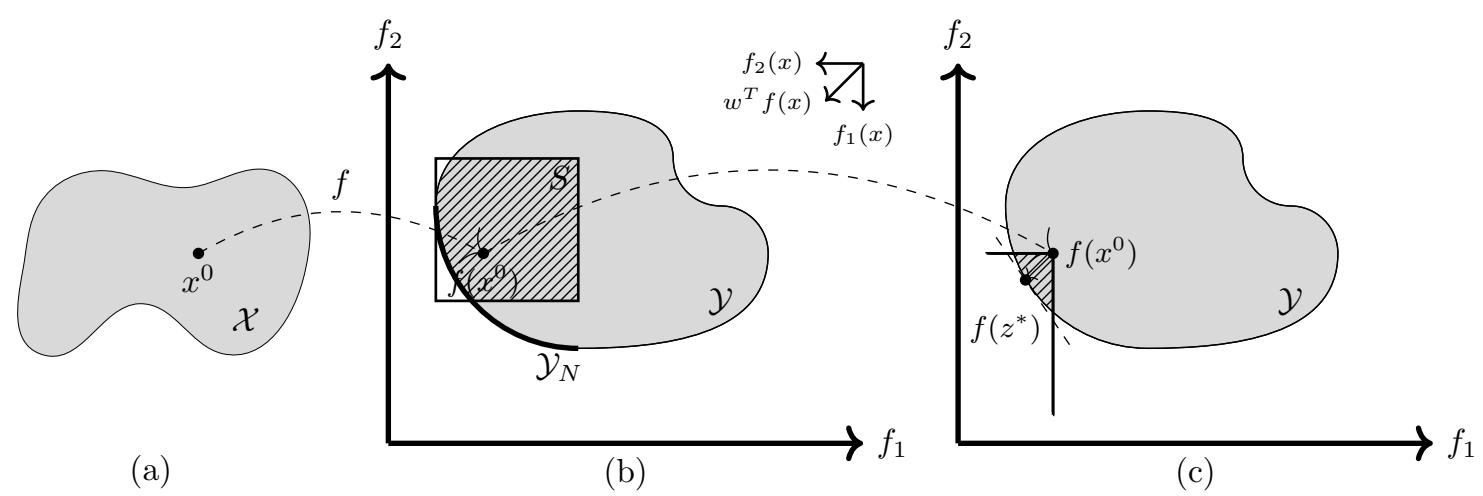

Figure 1: Upper and lower level problems of the BPP formulation.

In Figure 1, upper and lower level problems of the proposed BPP formulation are illustrated. The feasible region $\mathcal{X}$ in the decision space and its image in the outcome space $\mathcal{Y}$ are shown in Figure 1.a and 1.b, respectively. The nondominated set $\mathcal{Y}_{N}$ is marked on 1.b as bold and the given set $S \subseteq \mathbb{R}^{p}$ along with $\mathcal{Y}$ determines the feasible region of the upper level 
problem (region with dashed lines). For a given $x^{0} \in \mathcal{X}$ (Figure 1.a) such that $f\left(x^{0}\right) \in S$ (Figure 1.b), the feasible region of the lower level problem (region with dashed lines) is shown in Figure 1.c. For the $x^{0} \in \mathcal{X}$ and $w>0$ given in the figure, the rational reaction set has one element, $z^{*} \in \Omega\left(x^{0}\right)$ such that $f\left(z^{*}\right) \in \mathcal{Y}_{N}$ (Figure 1.c). In this case, $\left(x^{0}, z^{*}\right)$ is not an optimal solution to BPP since $d\left(f\left(x^{0}\right), f\left(z^{*}\right)\right)>0$ whereas for a nondominated solution that is in $S$ an upper level objective value of zero is possible.

The results above hold for a general MOP and an arbitrary closed set $S$. Hence, the bilevel formulation can be used to solve different MOP problems. In particular, an interactive use of BPP can be envisioned. In interactive methods, the Decision Maker is involved in the solution process and continuously interacts with the method to determine the most preferred solution [21]. The Decision Maker expresses preferences at each iteration in order to get efficient solutions that are of interest to her/him and learn what kind of solutions are attainable [34]. When the Decision Maker's preferences are expressed by using the set $S$, BPP is able to determine a nondominated solution that satisfies the Decision Maker's preferences. If there exists no such nondominated solution, BPP identifies it as well and the Decision Maker's preferences can be updated to rebuild $S$.

Before we proceed to discuss how BPP can be used to obtain a representation of the nondominated set of MOP when a set $S$ describing the Decision Maker's preferences is not readily available, we note that there are several issues surrounding solution of BPP for the general case. In addition to the known difficulty of solving bilevel problems in general [1], the structure of our problem may pose challenges. In particular, Slater's condition need not hold for some upper level feasible solutions because of the domination constraints in the lower level problem in BPP. As noted by Jahn in a single level study of the scalarization that corresponds to our lower level problem, when an efficient solution is present on the right hand side, the feasible set of the problem may become a singleton, thus violating Slater's condition [25]. In a bilevel setting, this may hurt the equivalence between a bilevel program with a convex lower level problem and a mathematical program with complementarity constraints as demonstrated in [10], rendering a Karush-Kuhn-Tucker (KKT) transformation ineffective. Several solution methods have been proposed to solve different types of bilevel programs with convex lower level problems such as the descent approach [48], bundle algorithm [9], penalty approach [32], trust-region method [6], smooth approximation of the KKT transformation [20] and reformulating the problem as a binary integer programming problem [19]. In addition, more complex bilevel programs have been studied such as problems with discrete variables [2, 49], with nonconvex lower level problems [35] and research on such problems is ongoing. BPP for MOLP can be solved with relatively well-established methods [51]. In Section 4, a KKT formulation reformulated with binary variables will be given for this case.

\section{Finding a Representation of the Nondominated Set}

In order to obtain a representation of the nondominated set with a specified coverage error, partitioning the image of the feasible region in the objective space into smaller sets and working on each partition element is a key aspect of our approach. BPP is used as a subproblem to examine whether the partition element contains any nondominated solutions or not. The quality of the representation is then improved via further partitioning, which 
is performed using a subdivision process in the spirit of those used in global optimization methods. We provide definitions about partition elements and their subdivision as well as definitions of representations and approximations. We then state the algorithm and discuss its validity and finiteness.

\subsection{Partition Elements and Their Subdivision}

An idea that has been used in branch and bound type global optimization approaches is to embed the feasible set into a simple polytope or a convex polyhedral set such as a rectangle, simplex or a polyhedral cone to facilitate computation of a bound for the original problem. This set is then broken into smaller pieces and the bounding operation is repeated [24]. We utilize this well-established infrastructure of partitioning to build a representation. We first define a partition.

Definition 2. Let $\mathcal{P}_{0}$ be a subset of $\mathbb{R}^{p}$ and $I$ be a finite subset of indices. A set $\left\{\mathcal{P}_{i}: i \in I\right\}$ of subsets of $\mathcal{P}_{0}$ is said to be a partition of $\mathcal{P}_{0}$ if $\mathcal{P}_{0}=\cup_{i \in I} \mathcal{P}_{i}, \mathcal{P}_{i} \cap \mathcal{P}_{k}=\partial \mathcal{P}_{i} \cap \partial \mathcal{P}_{k}$ for all $i, k \in I$ and $i \neq k$ where $\partial \mathcal{P}_{i}$ denotes the boundary of $\mathcal{P}_{i}$ [24].

A subdivision procedure is applied to a chosen partition element with the goal of replacing it with a finite number of newly formed partition elements. Let $\delta(\mathcal{P})$ denote the diameter of a partition element $\mathcal{P}$, which is determined as the maximum distance between two elements of $\mathcal{P}$, i.e. $\delta(\mathcal{P})=\sup _{y, \bar{y} \in \mathcal{P}} d(y, \bar{y})$. With this notation, exhaustiveness of a subdivision procedure is defined as follows.

Definition 3. A subdivision procedure is called exhaustive if $\lim _{i \rightarrow \infty} \delta\left(\mathcal{P}_{i}\right)=0$ for all nested subsequences $\left\{\mathcal{P}_{i}\right\}$ of partition elements generated by the subdivision [24].

The idea in our procedure is to start with an initial partition element $\mathcal{P}_{0}$ that contains $\mathcal{Y}$ and thus $\mathcal{Y}_{N}$. Then BPP is solved with this partition element. Then and thereafter, a current partition element will be subject to a subdivision procedure, i.e. it will be divided into smaller subsets in a way to yield partition elements of the same structure. Validity and finiteness of our approach rely on the exhaustiveness of the procedure.

\subsection{Representations}

Any finite discrete subset of the nondominated set is called a representation and is denoted as $\mathcal{Y}_{R}$. A representation should contain points from every portion of the nondominated set without missing any region. This is captured by the coverage error as defined in [41] and computed as

$$
\max _{y \in \mathcal{Y}_{N}} \min _{\bar{y} \in \mathcal{Y}_{R}} d(y, \bar{y})
$$

In (1), any point in the nondominated set $y \in \mathcal{Y}_{N}$ is represented by a closest point in the representation. The worst represented point in the nondominated set determines the coverage error associated with representation $\mathcal{Y}_{R}$. This measure is related to the one-sided Hausdorff distance between two sets $A$ and $B$ given by $d(A, B)=\sup _{x \in A} \inf _{y \in B} d(x, y)$ [23]. Under our assumptions of a compact $\mathcal{X}$ and continuous $f_{j}$ 's, the coverage error of 
representation $\mathcal{Y}_{R}$ is equivalent to the one-sided Hausdorff distance. Therefore from here on we use the notation $d\left(\mathcal{Y}_{N}, \mathcal{Y}_{R}\right)$ to denote the coverage error of representation $\mathcal{Y}_{R}$.

Our algorithm generates a representative set that conforms to a specified coverage error level $\alpha \in \mathbb{R}$. Note that although the definition of an $\alpha$-representation relies on $\mathcal{Y}_{N}$, the representation is generated and must conform to this standard in the absence of $\mathcal{Y}_{N}$.

Definition 4. A set $\mathcal{Y}_{R}$ is called an $\alpha$-representation of $\mathcal{Y}_{N}$ if $\left|\mathcal{Y}_{R}\right|<\infty, \mathcal{Y}_{R} \subseteq \mathcal{Y}_{N}$ and $d\left(\mathcal{Y}_{N}, \mathcal{Y}_{R}\right) \leq \alpha$.

Another concept in MOP is that of an approximation. Unlike representations, approximation sets may contain infinitely many solutions which are not necessarily nondominated but are within a certain proximity of the nondominated set. Approximate nondominated solutions are traditionally defined as feasible solutions that are not necessarily nondominated. These solutions usually become nondominated when subjected to some perturbation. The algorithm we are about to present generates an approximation that is consistent with the concept of $\epsilon$-efficiency in general and with a definition labeled as $E^{1}$ in [52] in particular. $E^{1}$ is a strong approximation that guarantees existence of an efficient point within a distance of $\epsilon$. The outcome version of $E^{1}$ can be defined as $\mathcal{Y}^{1}(\epsilon)=\left\{y \in \mathcal{Y}:\right.$ there exists $\bar{y} \in \mathcal{Y}_{N}$ such that $\left.d(y, \bar{y}) \leq \epsilon\right\} \quad$ [52]. We will later show that for a given $\alpha$, our approximation $\mathcal{Y}_{A}$ satisfies $\mathcal{Y}_{A} \subseteq \overline{\mathcal{Y}}^{1}(\alpha)$. although our approximate solutions are not guaranteed to belong to $\mathcal{Y}$ and can come from $\mathbb{R}^{p}$, they are guaranteed to conform with a given proximity requirement.

\subsection{The Representation Algorithm}

A $\mathcal{P}_{0}$ that covers $\mathcal{Y}$ can be built in accordance with the procedures used in global optimization methods developed for the particular type of partition element in use [24]. In the examples given in the next section, we illustrate one way of building $\mathcal{P}_{0}$ with rectangular partition elements. The algorithm initiates a search list that consists of $\mathcal{P}_{0}$ as the only partition element. In each iteration, the algorithm picks a partition element from the search list and solves BPP. If there exists an efficient solution whose image is in partition element $\mathcal{P}$, then the algorithm subdivides $\mathcal{P}$ into smaller partition elements. If there exists no nondominated solution in $\mathcal{P}$, the algorithm removes $\mathcal{P}$ from the search list. Since our aim is to obtain an $\alpha$-representation, we can eliminate some of the partition elements whose elements are within an $\alpha$ distance of the current representation. In other words, if $d\left(\mathcal{P}, \mathcal{Y}_{R}\right)$ is less than or equal to desired coverage error level $\alpha$, then further subdivision of $\mathcal{P}$ is not necessary and it can be eliminated from the search.

Finally, some of the partition elements can be eliminated by using dominance ideas. Let $\left(x^{*}, z^{*}\right)$ be an optimal solution of BPP. By Proposition $1, z^{*} \in \mathcal{X}_{E}$. Then the region $f\left(z^{*}\right)$ dominates and the region that consists of the points dominating $f\left(z^{*}\right)$ cannot contain any nondominated solutions. We define $R^{1}\left(f\left(z^{*}\right)\right)=\left\{y \in \mathbb{R}^{p}: y<f\left(z^{*}\right)\right\}$ and $R^{2}\left(f\left(z^{*}\right)\right)=$ $\left\{y \in \mathbb{R}^{p}: y>f\left(z^{*}\right)\right\}$. Hence, any partition element $\mathcal{P}$ with $\mathcal{P} \subseteq R^{1}\left(f\left(z^{*}\right)\right)$ or $\mathcal{P} \subseteq R^{2}\left(f\left(z^{*}\right)\right)$ can be eliminated.

The algorithm terminates when there exists no element in the list. We call this algorithm Representative Set Generation Algorithm (RSGA). A formal description of RSGA is given below. 


\section{Representative Set Generation Algorithm}

Input: Desired coverage error $(\alpha)$, MOP (objective functions $f_{j}, j \in\{1, \ldots, p\}$, feasible set $\mathcal{X}), \mathcal{P}_{0}$ that contains $\mathcal{Y}$, positive weight vector $w \in \mathbb{R}^{p}$.

Output: an $\alpha$-representation of the nondominated set $\left(\mathcal{Y}_{R}\right)$ and an approximation of the nondominated set $\left(\mathcal{Y}_{A}\right)$.

Step 0. $\mathcal{Y}_{R}=\emptyset, \mathcal{Y}_{A}=\emptyset$. Initialize the list $\mathcal{L}=\left\{\mathcal{P}_{0}\right\}$ where $\mathcal{Y} \subseteq \mathcal{P}_{0} . \mathrm{k}:=1$

Step k.1 If $\mathcal{L}$ is empty, return representative set $\mathcal{Y}_{R}$, approximation set $\mathcal{Y}_{A}$ and stop. Otherwise, pick a partition element from the list $\mathcal{L}$ with a largest diameter, i.e. $\mathcal{P}_{k} \in$ $\arg \max _{\mathcal{P} \in \mathcal{L}} \delta(\mathcal{P})$.

k.1.1. If there exists $y \in \mathcal{Y}_{R}$ such that $y \in \mathcal{P}_{k}$, then go to Step-k.2. Else, solve BPP with $\mathcal{P}_{k} \subseteq \mathbb{R}^{p}$ and $w>0$. If $\mathrm{BPP}$ is feasible, let $\left(x^{*}, z^{*}\right)$ denote an optimal solution. Otherwise, set $\mathcal{L}=\mathcal{L} \backslash\left\{\mathcal{P}_{k}\right\}$, k:=k+1. Go to Step k.1.

k.1.2. If $d\left(f\left(x^{*}\right), f\left(z^{*}\right)\right)=0$, then $\mathcal{Y}_{R}=\mathcal{Y}_{R} \cup\left\{f\left(x^{*}\right)\right\}$. Go to Step-k.2.

k.1.3. If $d\left(f\left(x^{*}\right), f\left(z^{*}\right)\right)>0$, then $\mathcal{L}=\mathcal{L} \backslash\left\{\mathcal{P}_{k}\right\}$. $\mathrm{k}:=\mathrm{k}+1$. Go to Step-k.1.

Step k.2. Construct a partition of $\mathcal{P}_{k}$. Let $\mathcal{L}_{k}^{\prime}$ be the collection of all new partition elements.

k.2.1. For each $\hat{\mathcal{P}} \in \mathcal{L}_{k}^{\prime}$, if $\hat{\mathcal{P}} \subseteq R^{1}(y)$ or $\hat{P} \subseteq R^{2}(y)$ for some $y \in \mathcal{Y}_{R}$, then $\mathcal{L}_{k}^{\prime}=\mathcal{L}_{k}^{\prime} \backslash\{\hat{\mathcal{P}}\}$.

k.2.2. For each $\hat{\mathcal{P}} \in \mathcal{L}_{k}^{\prime}$, if $d\left(\hat{\mathcal{P}}, \mathcal{Y}_{R}\right) \leq \alpha$, then $\mathcal{L}_{k}^{\prime}=\mathcal{L}_{k}^{\prime} \backslash\{\hat{\mathcal{P}}\}$ and $\mathcal{Y}_{A}=\mathcal{Y}_{A} \cup\{\hat{\mathcal{P}}\}$.

Set $\mathcal{L}=\left(\mathcal{L} \backslash\left\{\mathcal{P}_{k}\right\}\right) \cup \mathcal{L}_{k}^{\prime}, \mathrm{k}:=\mathrm{k}+1$. Go to Step k.1.

RSGA starts to search the outcome space with a sufficiently large set $\mathcal{P}_{0}$ and iteratively refines the initial partition by obtaining new nondominated solutions. Some of the subsets of the initial search space are removed if either there exists no nondominated solution in the partition element or the partition element satisfies the acceptable coverage error level $\alpha$. Note that if $\mathcal{Y}_{N}=\emptyset$ RSGA will terminate during its first iteration returning $\mathcal{Y}_{R}=\emptyset$. In what follows, we assume that $\mathcal{Y}_{N} \neq \emptyset$. For most of the results below, the subdivision procedure used in RSGA must be exhaustive. Therefore we also assume that an exhaustive subdivision procedure is employed.

Lemma 2. Suppose $\alpha=0$ in RSGA. Let $\delta_{k}$ denote the diameter of the partition element picked at step $k$ of $R S G A$, i.e. $\delta_{k}=\delta\left(\mathcal{P}_{k}\right)=\max _{\mathcal{P} \in \mathcal{L}} \delta(\mathcal{P})$. Then $\left\{\delta_{k}\right\}$ is an infinite sequence and $\lim _{k \rightarrow \infty} \delta_{k}=0$.

Proof. First, observe that the algorithm stops when the list of partition elements becomes empty. A partition element $\mathcal{P}$ is discarded in step $k .1 .1$ when $\mathcal{P} \cap \mathcal{Y}=\emptyset$, in step $k .1 .3$ or $k .2 .1$ when $\mathcal{P} \cap \mathcal{Y}_{N}=\emptyset$. Hence a partition element $\mathcal{P}$ with $\mathcal{P} \cap \mathcal{Y}_{N} \neq \emptyset$ can only be discarded in step $k .2 .2$, which implies with $\alpha=0$ such partition elements cannot be eliminated and remain in the list $\mathcal{L}$. Thus RSGA generates an infinite sequence $\delta_{k}$. Next, observe that $\delta_{k}$ is a nonincreasing sequence bounded from below and must converge. Since an exhaustive subdivision procedure is used, there exists a nested subsequence of $\left\{\mathcal{P}_{k}\right\}$ such that $\lim _{i \rightarrow \infty} \delta\left(\mathcal{P}_{k_{i}}\right)=0$. Thus $\lim _{k \rightarrow \infty} \delta_{k}=\delta\left(\mathcal{P}_{k}\right)=0$ follows.

Theorem 2. Suppose $\alpha=0$ in RSGA. Let $\mathcal{Y}_{R}^{k}$ denote the representation as of iteration $k$ of the algorithm. Then $\lim _{k \rightarrow \infty} \mathcal{Y}_{R}^{k}=\mathcal{Y}_{N}$, where convergence is in the Hausdorff sense. 
Proof. Let $\alpha^{k}=d\left(\mathcal{Y}_{N}, \mathcal{Y}_{R}^{k}\right)=\max _{y \in \mathcal{Y}_{N}} \min _{\bar{y} \in \mathcal{Y}_{R}^{k}} d(y, \bar{y})$. Observe that $\alpha^{k} \geq 0$ for all $k$ and $\alpha^{k} \leq \alpha^{k-1}$ since $\mathcal{Y}_{R}^{k} \supseteq \mathcal{Y}_{R}^{k-1}$. Now, let $y^{0} \in \mathcal{Y}_{N}$ be an arbitrary point. Then $y^{0} \in \mathcal{P}_{0}$. As discussed in proof of Lemma 2, a partition element that contains $y^{0}$ can only be eliminated in step $k .2 .2$ when it satisfies the coverage condition. This implies that with $\alpha=0$, such partition elements cannot be eliminated. Therefore a nested sequence of partition elements $\overline{\mathcal{P}}_{0} \supseteq \overline{\mathcal{P}}_{1} \supseteq \ldots \supseteq \overline{\mathcal{P}}_{i} \supseteq \ldots$ are generated throughout the algorithm such that $y^{0} \in \overline{\mathcal{P}}_{i}$ for all $i$. Moreover, $\lim _{i \rightarrow \infty} \delta\left(\overline{\mathcal{P}}_{i}\right)=0$ since an exhaustive subdivision procedure is used. This implies, for any $\epsilon>0, \exists N_{\epsilon}$ such that for all $i \geq N_{\epsilon}, \max _{y, \bar{y} \in \overline{\mathcal{P}}_{i}} d(y, \bar{y})<\epsilon$. Pick $\hat{\imath}>N_{\epsilon}$. Then $\max _{\bar{y} \in \overline{\mathcal{P}}_{\hat{\imath}}} d\left(y^{0}, \bar{y}\right)<\epsilon$. Since $\lim _{k \rightarrow \infty} \delta_{k}=0$ by Lemma 2, RSGA will set $\mathcal{P}_{\hat{k}}=\overline{\mathcal{P}}_{\hat{\imath}}$ at some iteration $\hat{k}$, obtain $y^{*} \in \mathcal{Y}_{N} \cap \mathcal{P}_{\hat{k}}$ and set $\mathcal{Y}_{R}^{\hat{k}}=\mathcal{Y}_{R}^{\hat{k}-1} \cup\left\{y^{*}\right\}$. Then $\min _{\bar{y} \in \mathcal{Y}_{R}^{\hat{k}}} d\left(y^{0}, \bar{y}\right)<\epsilon$ follows.

Since $y^{0} \in \mathcal{Y}_{N}$ is arbitrary, it can be stated that for any $\epsilon>0$, there exists $N$ such that for all $k \geq N, d\left(\mathcal{Y}_{N}, \mathcal{Y}_{R}^{k}\right)<\epsilon$, which implies $\lim _{k \rightarrow \infty} d\left(\mathcal{Y}_{N}, \mathcal{Y}_{R}^{k}\right)=0$. Now, observe the following. Since $\mathcal{Y}_{R}^{k} \subseteq \mathcal{Y}_{N}, d\left(\mathcal{Y}_{R}^{k}, \mathcal{Y}_{N}\right)=0$ for all $k$. Therefore the Hausdorff distance between $\mathcal{Y}_{N}$ and $\mathcal{Y}_{R}^{k}$, which is given by $d^{H}\left(\mathcal{Y}_{N}, \mathcal{Y}_{R}^{k}\right)=\max \left\{d\left(\mathcal{Y}_{N}, \mathcal{Y}_{R}^{k}\right), d\left(\mathcal{Y}_{R}^{k}, \mathcal{Y}_{N}\right)\right\}$ approaches zero as $k \rightarrow \infty$, which implies $\lim _{k \rightarrow \infty} \mathcal{Y}_{R}^{k}=\mathcal{Y}_{N}[39]$.

Corollary 1. Suppose $\alpha>0$ in RSGA. Then RSGA is finite and $\mathcal{Y}_{R}$ is an $\alpha$-representation of $\mathcal{Y}_{N}$.

Proof. From Lemma 2, for any $\alpha>0$, there exists an iteration $\hat{k}$ such that $\delta_{\hat{k}}$ becomes less than $\alpha$. We will argue that $\mathcal{P}_{\hat{k}}$ will be eliminated from $\mathcal{L}$, even if $\mathcal{P}_{\hat{k}} \cap \mathcal{Y}_{N} \neq \emptyset$ (otherwise $\mathcal{P}_{\hat{k}}$ is clearly eliminated as discussed before). If RSGA does not detect a $y^{*} \in \mathcal{P}_{\hat{k}} \cap \mathcal{Y}_{R}$, then BPP will be solved and $y^{*} \in \mathcal{P}_{\hat{k}}$ will be added to $\mathcal{Y}_{R}$. Thus when step $k .2$ is executed, for any subset $\hat{\mathcal{P}}$ of $\mathcal{P}_{\hat{k}}, d\left(\hat{\mathcal{P}}, \mathcal{Y}_{R}\right) \leq \alpha$ will hold, leading to their elimination. This proves that RSGA is finite, and for any partition element with nondominated points at elimination, the coverage error requirement is met.

Corollary 2. Suppose $\alpha>0$ in $R S G A$. The approximation $\mathcal{Y}_{A}$ delivered by $R S G A$ contains $\mathcal{Y}_{N}$. Moreover, $\mathcal{Y}_{A} \subseteq \overline{\mathcal{Y}}^{1}(\alpha)=\left\{y \in \mathbb{R}^{p}\right.$ : there exists $\bar{y} \in \mathcal{Y}_{N}$ such that $\left.d(y, \bar{y}) \leq \alpha\right\}$.

Proof. Partition elements that contain nondominated solutions can only be eliminated in $k .2 .2$ and whenever they are eliminated from the search list, they are added to $\mathcal{Y}_{A}$. Therefore $\mathcal{Y}_{N} \subseteq \mathcal{Y}_{A}$ follows. Moreover, any $y \in \mathcal{Y}_{A}$ belongs to a partition $\mathcal{P}$ such that $d\left(\mathcal{P}, \mathcal{Y}_{R}\right) \leq \alpha$. Thus for any $y \in \mathcal{Y}_{A}$, there exists $y^{*} \in \mathcal{Y}_{N}$ such that $d\left(y, y^{*}\right) \leq \alpha$.

Note that we do not have $\mathcal{Y}_{A} \subseteq \mathcal{Y}^{1}(\alpha)$, as our partition elements may contain points that are not in $\mathcal{Y}$.

\section{Illustrative Examples}

In this section, we illustrate the proposed method on two MOLP problems. A general MOLP problem can be formulated as follows,

$$
\min \{C x: A x \leq b, x \geq 0\}
$$


Above, $C \in \mathbb{R}^{p \times n}$ represents the objective coefficients, $A \in \mathbb{R}^{m \times n}$ is the matrix of technical coefficients, $b \in \mathbb{R}^{m}$ is the right hand side vector, and $x \in \mathbb{R}^{n}$ is the vector of decision variables. We define $c^{\prime}=C^{T} w$ and let $S=\left\{y \in \mathbb{R}^{p}: l \leq y \leq u\right\}$. $S$ is a rectangle with lower vertex $l \in \mathbb{R}^{p}$ and upper vertex $u \in \mathbb{R}^{p}$. The vertices may be indicated by a Decision Maker as specifications on objective function values or may be determined so as to describe $\mathcal{P}_{0}$ as we will discuss shortly. With this notation, BPP can be expressed as follows.

$$
\begin{array}{cl}
\min _{x \geq 0} & f^{U}(x, z)=d(C x, C z) \\
\text { s.t. } & A x \leq b \\
& l \leq C x \leq u \\
& \min _{z \geq 0} \quad f^{L}(z)=c^{T} z \\
& \text { s.t. } \quad A z \leq b \\
& C z \leq C x .
\end{array}
$$

We will reformulate it as a single-level integer programming problem [19]. This approach utilizes KKT optimality conditions for the lower level problem to turn BPP into a single level optimization problem [11] and manages complementarity constraints via binary variables. Let $\omega \in \mathbb{R}^{m}, \pi \in \mathbb{R}^{n}$ and $\mu \in \mathbb{R}^{p}$ be the dual variables associated with $A z \leq b, z \geq 0$ and $C z \leq C x$, respectively. For the complementary slackness condition, three sets of binary variables, $o \in\{0,1\}^{m}, t \in\{0,1\}^{n}$ and $v \in\{0,1\}^{p}$, are defined. Let $M$ be a sufficiently large number, and $I$ denote the $n \times n$ identity matrix. Our formulation is given by

$$
\begin{array}{ll}
\min & d(C x, C z) \\
\text { s.t. } & A x \leq b \\
& l \leq C x \leq u \\
& c^{\prime}+A^{T} \omega-I \pi+C^{T} \mu=0 \\
& A z-b \leq 0 \\
& C z-C x \leq 0 \\
& A z-b \geq-M(\mathbf{1}-o) \\
& z \geq-M(\mathbf{1}-t) \\
& c z-c x \geq-M(\mathbf{1}-v) \\
& \omega \leq M o \\
& \pi \leq M t \\
& \mu \leq M v \\
& x, z, \omega, \pi, \mu \geq 0 \\
& o \in\{0,1\}^{m} \\
& t \in\{0,1\}^{n} \\
& v \in\{0,1\}^{p} .
\end{array}
$$

This formulation can be linearized if the $\ell_{1}$-norm or the $\ell_{\infty}$-norm is used in the objective function following the procedure given in [41]. Below, we will give two examples where we use the $\ell_{1}$-norm. In both examples, we compute representations and approximations with 
different quality specifications. We define the initial rectangle $\mathcal{P}_{0}$ as $\mathcal{P}_{0}=\left\{y \in \mathbb{R}^{p}: y^{I} \leq\right.$ $\left.y \leq y^{U}\right\}$ where $y_{j}^{I}=\min _{x \in \mathcal{X}} f_{j}(x)$ and $y_{j}^{U}=\max _{x \in \mathcal{X}} f_{j}(x)$. We employ bisection using a longest edge for subdivision, which is an exhaustive process [24]. The weights of the lower level problem are set to $1, w=\mathbf{1}$.

Our first MOLP problem has $n=p=2$ with the following parameters given below [13].

$$
C=\left[\begin{array}{rr}
3 & 1 \\
-1 & -2
\end{array}\right], A=\left[\begin{array}{rr}
0 & 1 \\
3 & -1
\end{array}\right], b=\left[\begin{array}{l}
3 \\
6
\end{array}\right] .
$$

Outcome space and the nondominated set (bold faces of the two-dimensional polytope) are shown in Figure 2. To compute the coverage error, we use the $\ell_{\infty}$-norm normalized with respect to objective value ranges. In Figure 2, representative sets obtained by RSGA with three different $\alpha$ levels of $0.05,0.10$ and 0.20 are given. The cardinality of representative sets are 30,17 , and 8, respectively. RSGA solves 45, 24, and 14 subproblems until termination. In the same figure, approximations of the nondominated set obtained by RSGA with the three $\alpha$ levels are given.

We compute the exact coverage errors associated with these representations using the fact that the nondominated set of the problem is a union of nondominated faces, which turn out to be 0.0313 for $\alpha=0.05,0.0625$ for $\alpha=0.10$, and 0.1667 for $\alpha=0.20$. In Figure 2, we also mark the worst represented point of the nondominated set with a ".$+ "$

The second problem has $n=p=3$ and $C=-I[3]$.

$$
A=\left[\begin{array}{rrr}
6 & 15 & 10 \\
5 & 8 & 12 \\
22 & 29 & 28 \\
24 & 16 & 11 \\
1 & 0 & 4 \\
8 & 0 & 1
\end{array}\right], b=\left[\begin{array}{c}
210 \\
152 \\
458 \\
312 \\
40 \\
72
\end{array}\right]
$$

To make interpreting the three-dimensional figures easier, we illustrate the problem as a maximization problem therefore multiplying each component of the results by -1 . The feasible set in outcome space and the nondominated set (shaded faces of the polytope) are shown in Figure 3. In this figure, representative sets with $0.05,0.10$ and 0.20 coverage errors are given. The cardinality of representative sets are 585, 164, and 43, respectively. RSGA solves 1026, 278, and 72 subproblems until termination. In Figure 4, approximations of the nondominated set obtained by RSGA with the three $\alpha$ levels are given. The exact coverage errors associated with these representations are 0.0495 for $\alpha=0.05,0.0778$ for $\alpha=0.10$, and 0.1477 for $\alpha=0.20$. Again, we show the worst represented point of the nondominated set with a "+" marker.

On sample MOLPs, we observe that representations are well-dispersed over the nondominated set (uniformity), and the number of nondominated solutions (cardinality) in the representative set decreases significantly when we double the allowed coverage error level although the procedure does not have direct control over these measures. 


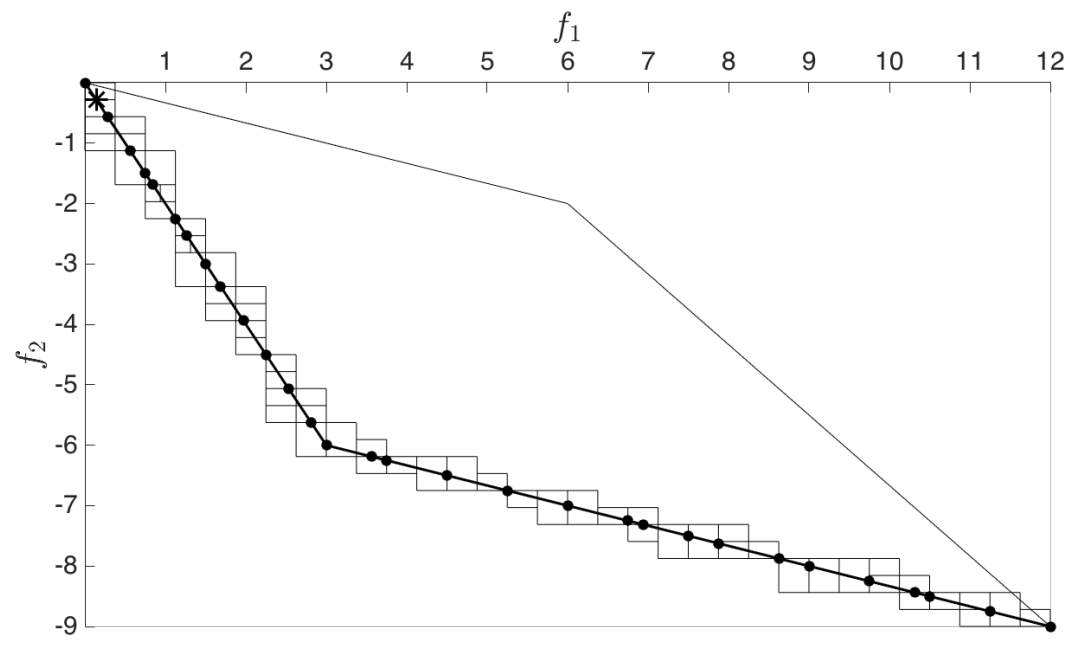

(a) $\alpha=0.05$

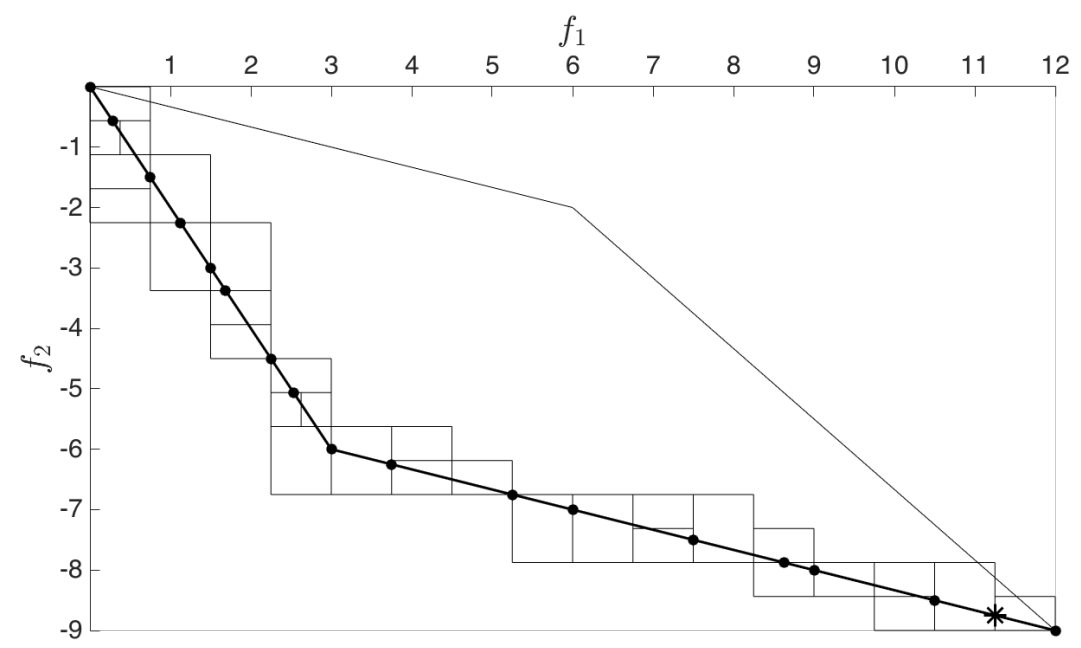

(b) $\alpha=0.10$

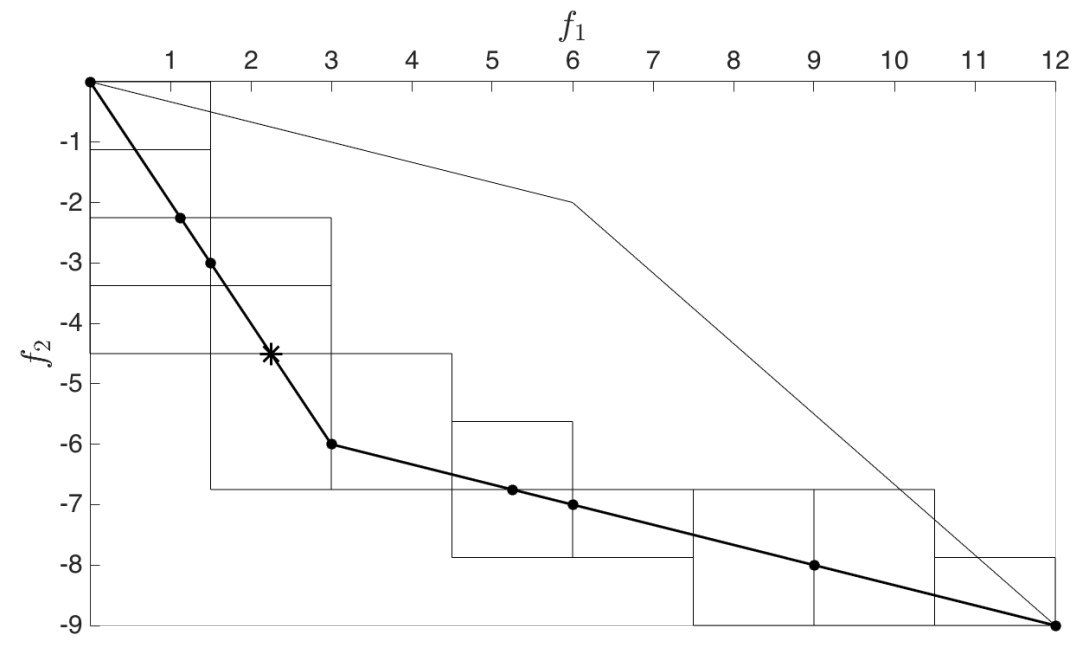

(c) $\alpha=0.20$

Figure 2: Representative and approximation sets, and the worst representative points of the nondominated sets with different coverage error factors for the biobjective example. 


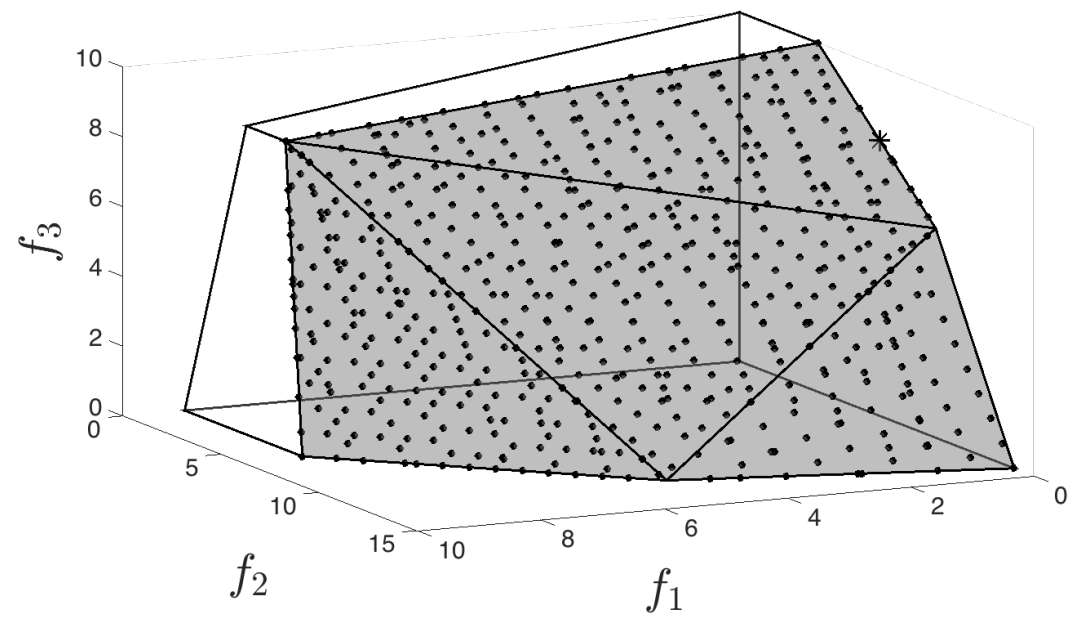

(a) $\alpha=0.05$

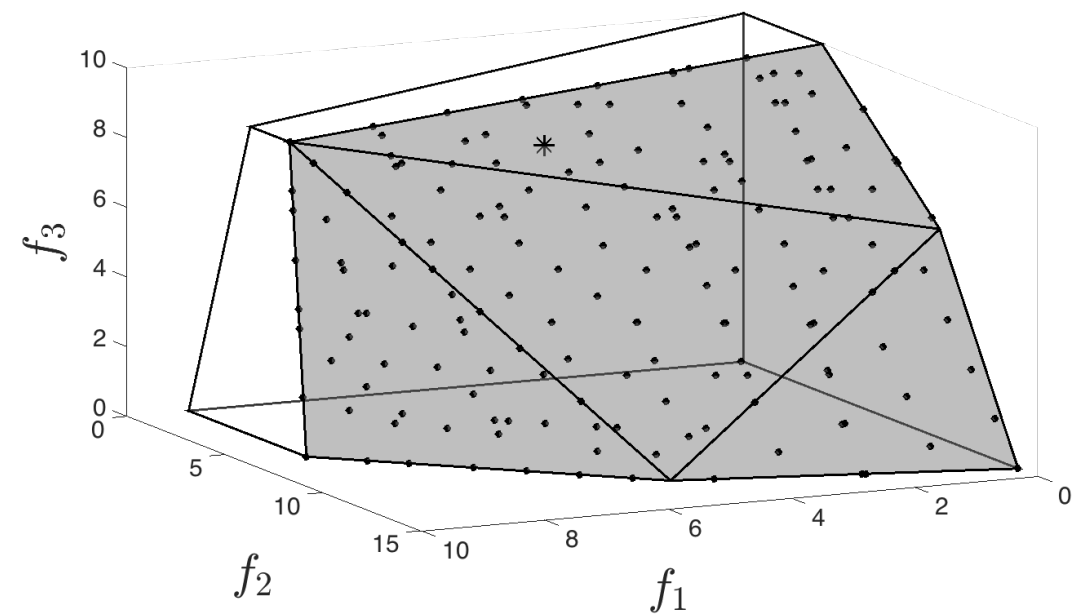

(b) $\alpha=0.10$

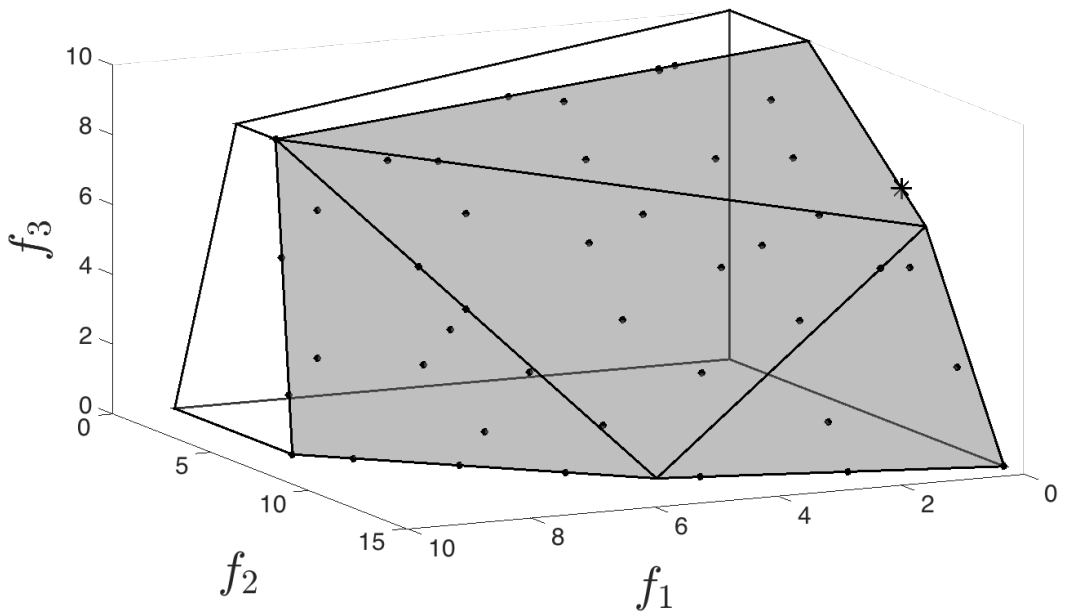

(c) $\alpha=0.20$

Figure 3: Representative sets and worst representative points of the nondominated sets with different coverage error factors. 


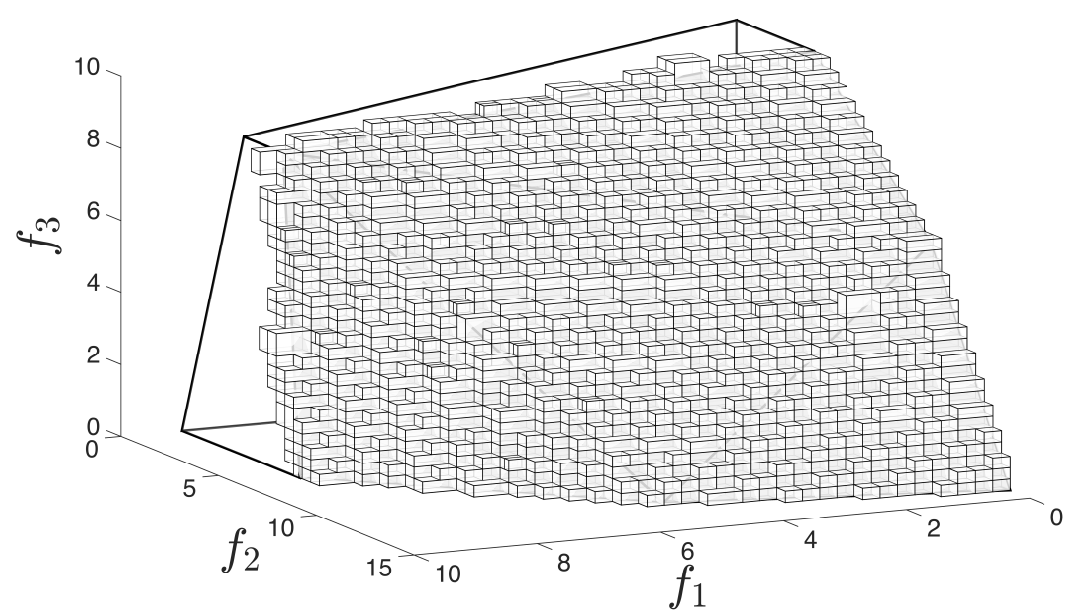

(a) $\alpha=0.05$

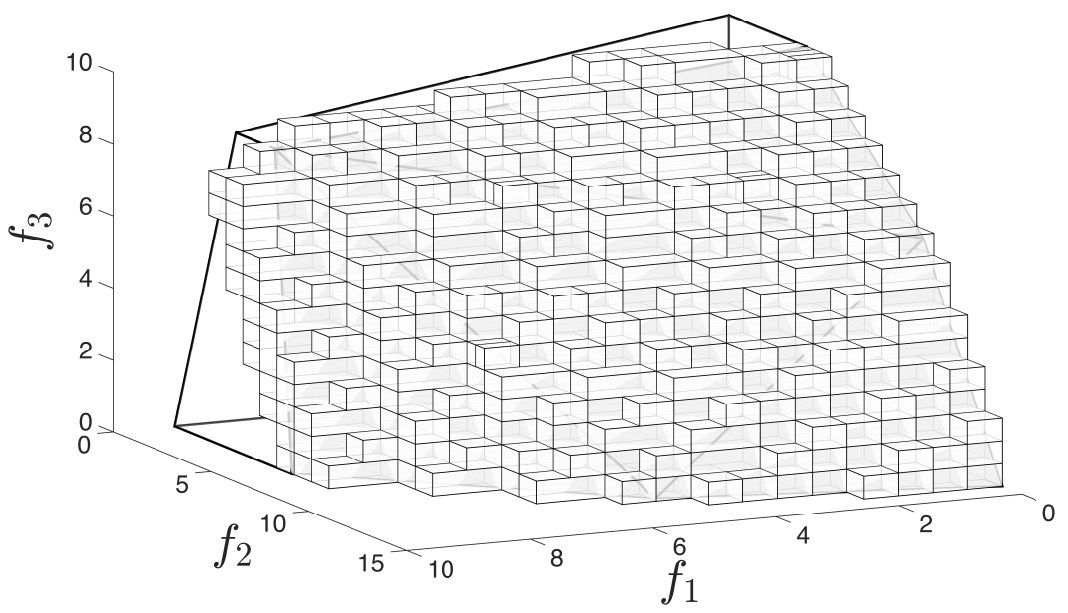

(b) $\alpha=0.10$

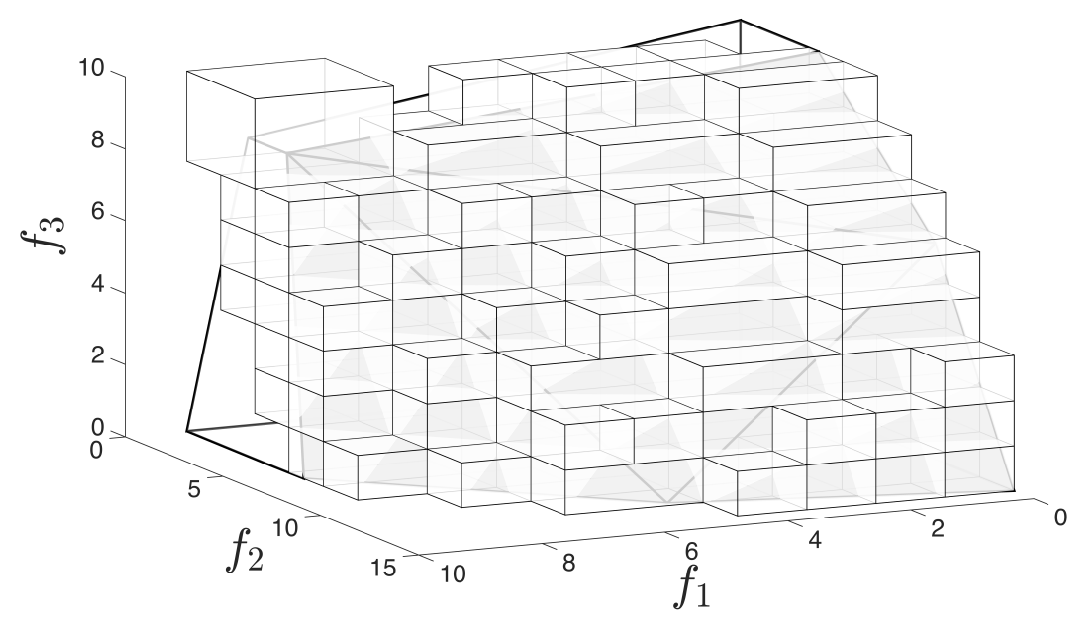

(c) $\alpha=0.20$

Figure 4: Approximation of the nondominated set with union of rectangles. 


\section{Conclusions and Future Work}

We propose a bilevel programming formulation that can identify nondominated solutions in a targeted region associated with an MOP. We suggest that the formulation may be used in an interactive way if preferences of the Decision Maker can help express the region in terms of constraints. When this is not possible, we suggest the formulation can be embedded into a search framework as those used in global optimization and a representation of the nondominated set that conforms to a quality specification in terms of coverage error can be obtained as well as an approximation with certain properties. We show that the representation algorithm is finite when a positive quality level is chosen and the representation delivered converges to the nondominated set when the quality level is chosen as zero.

There are several paths to follow for further research. An immediate extension might explore interactive use of BPP. In particular, translating the Decision Maker's preferences into building the set $S$ in a good way requires further research. Although our lower level problem incorporates weights, the upper level problem optimizes distances in the objective space and is indifferent among nondominated solutions in $S$ when several such solutions exist. It is possible to use a metric that involves weights in the upper level objective function. Whether this would guide the search in a desired direction requires experimentation.

Our approach for finding representations and approximations is presented in a standard way here and several variations may be possible. Choice of different partition elements and construction of a tighter initial one may be worth exploring. Stronger dominance elimination techniques might be sought. Alternative search strategies other than largest diameter first might be investigated.

It may be possible to use BPP for finding the nadir point of MOP. The nadir point is constructed from the worst objective function values over the efficient set and determination of the nadir point is generally a hard problem [14]. To obtain the nadir value for one of the objective functions, for instance $f_{1}$, BPP can be utilized to conduct a line search between a lower bound on the nadir value, for instance obtained via the pay-off table, and an upper bound on the nadir value, for instance the associated component of the ideal solution. Initially $S$ can be chosen so as to contain all nondominated solutions and can be constrained on values of $f_{1}$ only in order to facilitate a desired line search. Whether such an approach is computationally competitive requires further investigation.

$\mathrm{BPP}$ is a subproblem with strong theoretical aspects and serious numerical limitations. Computational and numerical challenges associated with solving BPPs with different structures may be studied, including an assessment of the impact and implications of solving the bilevel programming subproblem heuristically. As we build upon bilevel programming and global optimization, implications of recent research findings in these fields on solving BPP

may be explored. Clearly, alternative subproblems with equivalent theoretical and better numerical aspects can be sought.

\section{References}

[1] Bard, J.: Some properties of the bilevel programming problem. Journal of Optimization Theory and Applications 68(2), 371-378 (1991) 
[2] Bard, J.F., Moore, J.T.: An algorithm for the discrete bilevel programming problem. Naval Research Logistics (NRL) 39(3), 419-435 (1992)

[3] Benson, H.P., Sayin, S.: Towards finding global representations of the efficient set in multiple objective mathematical programming. Naval Research Logistics 44(1), 47-67 (1997)

[4] Benson, H.P., Sun, E.: A weight set decomposition algorithm for finding all efficient extreme points in the outcome set of a multiple objective linear program. European Journal of Operational Research 139(1), 26-41 (2002)

[5] Bokrantz, R., Forsgren, A.: An algorithm for approximating convex Pareto surfaces based on dual techniques. INFORMS Journal on Computing 25(2), 377-393 (2013)

[6] Colson, B., Marcotte, P., Savard, G.: A trust-region method for nonlinear bilevel programming: algorithm and computational experience. Computational Optimization and Applications 30(3), 211-227 (2005)

[7] Dächert, K., Klamroth, K.: A linear bound on the number of scalarizations needed to solve discrete tricriteria optimization problems. Journal of Global Optimization 61(4), 643-676 (2015)

[8] Das, I., Dennis, J.E.: Normal-boundary intersection: A new method for generating the Pareto surface in nonlinear multicriteria optimization problems. SIAM Journal on Optimization 8(3), 631-657 (1998)

[9] Dempe, S.: A bundle algorithm applied to bilevel programming problems with nonunique lower level solutions. Computational Optimization and Applications 15(2), 145-166 (2000)

[10] Dempe, S., Dutta, J.: Is bilevel programming a special case of a mathematical program with complementarity constraints? Mathematical Programming 131(1-2), 37-48 (2012)

[11] Dempe, S., Zemkoho, A.B.: On the Karush-Kuhn-Tucker reformulation of the bilevel optimization problem. Nonlinear Analysis: Theory, Methods \& Applications 75(3), $1202-1218$ (2012)

[12] Ecker, J.G., Kouada, I.A.: Finding all efficient extreme points for multiple objective linear programs. Mathematical Programming 14(1), 249-261 (1978)

[13] Ehrgott, M.: Multicriteria Optimization. Springer, Berlin (2005)

[14] Ehrgott, M., Tenfelde-Podehl, D.: Computation of ideal and nadir values and implications for their use in MCDM methods. European Journal of Operational Research 151(1), 119-139 (2003)

[15] Eichfelder, G.: An adaptive scalarization method in multiobjective optimization. SIAM Journal on Optimization 19(4), 1694-1718 (2009) 
[16] Eichfelder, G.: A constraint method in nonlinear multi-objective optimization. In: Multiobjective Programming and Goal Programming, pp. 3-12. Springer (2009)

[17] Faulkenberg, S.L., Wiecek, M.M.: On the quality of discrete representations in multiple objective programming. Optimization and Engineering 11(3), 423-440 (2010)

[18] Faulkenberg, S.L., Wiecek, M.M.: Generating equidistant representations in biobjective programming. Computational Optimization and Applications 51(3), 1173-1210 (2012)

[19] Fortuny-Amat, J., McCarl, B.: A representation and economic interpretation of a twolevel programming problem. The Journal of the Operational Research Society 32(9), 783-792 (1981)

[20] Fukushima, M., Pang, J.S.: Convergence of a smoothing continuation method for mathematical progams with complementarity constraints. In: Ill-posed Variational Problems and Regularization Techniques, pp. 99-110. Springer (1999)

[21] Geoffrion, A.M., Dyer, J.S., Feinberg, A.: An interactive approach for multi-criterion optimization, with an application to the operation of an academic department. Management Science 19(4), 357-368 (1972)

[22] Hamacher, H.W., Pedersen, C.R., Ruzika, S.: Finding representative systems for discrete bicriterion optimization problems. Operations Research Letters 35(3), 336-344 (2007)

[23] Hausdorff, F.: Set Theory. Chelsea Publ. Co., New York (1962)

[24] Horst, R., Tuy, H.: Global Optimization: Deterministic Approaches. Springer (1990)

[25] Jahn, J.: Vector Optimization. Springer (2009)

[26] Karasakal, E., Köksalan, M.: Generating a representative subset of the nondominated frontier in multiple criteria decision making. Operations Research 57(1), 187-199 (2009)

[27] Kim, I., De Weck, O.: Adaptive weighted-sum method for bi-objective optimization: Pareto front generation. Structural and Multidisciplinary Optimization 29(2), 149-158 (2005)

[28] Kim, I.Y., de Weck, O.L.: Adaptive weighted sum method for multiobjective optimization: a new method for Pareto front generation. Structural and Multidisciplinary Optimization 31(2), 105-116 (2006)

[29] Kirlik, G., Sayın, S.: A new algorithm for generating all nondominated solutions of multiobjective discrete optimization problems. European Journal of Operational Research 232(3), 479-488 (2014)

[30] Kouvelis, P., Saym, S.: Algorithm robust for the bicriteria discrete optimization problem. Annals of Operations Research 147(1), 71-85 (2006) 
[31] Leyffer, S.: A complementarity constraint formulation of convex multiobjective optimization problems. INFORMS Journal on Computing 21(2), 257-267 (2009)

[32] Marcotte, P., Zhu, D.L.: Exact and inexact penalty methods for the generalized bilevel programming problem. Mathematical Programming 74(2), 141-157 (1996)

[33] Masin, M., Bukchin, Y.: Diversity maximization approach for multiobjective optimization. Operations Research 56(2), 411-424 (2008)

[34] Miettinen, K.: Nonlinear Multiobjective Optimization, vol. 12. Springer (1999)

[35] Mitsos, A., Lemonidis, P., Barton, P.I.: Global solution of bilevel programs with a nonconvex inner program. Journal of Global Optimization 42(4), 475-513 (2008)

[36] Özpeynirci, O., Köksalan, M.: An exact algorithm for finding extreme supported nondominated points of multiobjective mixed integer programs. Management Science 56(12), 2302-2315 (2010)

[37] Pereyra, V., Saunders, M., Castillo, J.: Equispaced Pareto front construction for constrained bi-objective optimization. Mathematical and Computer Modelling 57(9), 2122-2131 (2013)

[38] Przybylski, A., Gandibleux, X., Ehrgott, M.: A two phase method for multi-objective integer programming and its application to the assignment problem with three objectives. Discrete Optimization 7(3), 149-165 (2010)

[39] Rockafellar, R.T., Wets, R.J.B.: Variational Analysis, vol. 317. Springer (1998)

[40] Sayı, S.: An algorithm based on facial decomposition for finding the efficient set in multiple objective linear programming. Operations Research Letters 19(2), 87-94 (1996)

[41] Sayın, S.: Measuring the quality of discrete representations of efficient sets in multiple objective mathematical programming. Mathematical Programming 87(3), 543-560 (2000)

[42] Saym, S.: A procedure to find discrete representations of the efficient set with specified coverage errors. Operations Research pp. 427-436 (2003)

[43] Sayın, S., Kouvelis, P.: The multiobjective discrete optimization problem: A weighted min-max two-stage optimization approach and a bicriteria algorithm. Management Science 51(10), 1572-1581 (2005)

[44] Shao, L., Ehrgott, M.: Discrete representation of non-dominated sets in multi-objective linear programming. European Journal of Operational Research (2016)

[45] Stidsen, T., Andersen, K.A., Dammann, B.: A branch and bound algorithm for a class of biobjective mixed integer programs. Management Science 60(4), 1009-1032 (2014)

[46] Sylva, J., Crema, A.: A method for finding well-dispersed subsets of non-dominated vectors for multiple objective mixed integer linear programs. European Journal of Operational Research 180(3), 1011-1027 (2007) 
[47] Vaz, D., Paquete, L., Fonseca, C.M., Klamroth, K., Stiglmayr, M.: Representation of the non-dominated set in biobjective discrete optimization. Computers \& Operations Research 63, 172-186 (2015)

[48] Vicente, L., Savard, G., Júdice, J.: Descent approaches for quadratic bilevel programming. Journal of Optimization Theory and Applications 81(2), 379-399 (1994)

[49] Vicente, L., Savard, G., Judice, J.: Discrete linear bilevel programming problem. Journal of Optimization Theory and Applications 89(3), 597-614 (1996)

[50] Vincent, T., Seipp, F., Ruzika, S., Przybylski, A., Gandibleux, X.: Multiple objective branch and bound for mixed 0-1 linear programming: Corrections and improvements for the biobjective case. Computers \& Operations Research 40(1), 498-509 (2013)

[51] Wen, U.P., Hsu, S.T.: Linear bi-level programming problems - a review. Journal of the Operational Research Society 42(2), 125-133 (1991)

[52] White, D.J.: Epsilon efficiency. Journal of Optimization Theory and Applications 49(2), 319-337 (1986)

[53] Yu, P.L., Zeleny, M.: The set of all nondominated solutions in linear cases and a multicriteria simplex method. Journal of Mathematical Analysis and Applications 49(2), 430-468 (1975) 